\title{
Mudança na produtividade dos fatores de produção da cafeicultura nas principais regióes produtoras do Brasil ${ }^{1}$
}

\author{
Marília F. Maciel Gomes ${ }^{2}$ \\ Patrícia Lopes Rosado ${ }^{3}$
}

Resumo - Neste trabalho, estudam-se a transformação no comportamento produtivo da cafeicultura e as relações existentes no mercado de fatores nos Estados de Minas Gerais e São Paulo, no período de 1975 a 2001. Especificamente, avalia-se a sensibilidade do uso dos insumos a alterações nos preços, assim como a interdependência no mercado dos fatores produtivos. Para tanto, utilizou-se a elasticidade de substituição de Allen. As despesas com insumos e mão-de-obra apresentaram as maiores parcelas dos custos operacionais unitários de produção em Minas Gerais e São Paulo. Em relação à substitutibilidade/complementaridade entre os fatores em Minas Gerais, nota-se que havia relação de complementaridade entre aluguel da terra e operações com máquinas, terra e mão-de-obra, e entre máquinas e insumos, e relação de substitutibilidade entre os demais fatores. Já no Estado de São Paulo, observa-se que os fatores aluguel da terra e mão-de-obra foram complementares e os demais substitutos entre si.

\footnotetext{
${ }^{1}$ As autoras agradecem aos professores Erly Cardoso Teixeira e João Eustáquio de Lima e aos revisores da SOBER pelas sugestões.

${ }^{2}$ Professora doutora do Departamento de Economia Rural, DER -UFV. Bolsista CNPq. mfmogmes@ufv.br

${ }_{3}^{3}$ Doutoranda do Departamento de Economia Rural, DER -UFV. patriciarosado@yahoo. com.br
} 
Palavras-chaves: Custo, translog, elasticidade de substituição de Allen, café.

Classificação JEL: D24.

\begin{abstract}
This paper aimed to study the production behavior of the coffee production and the existing relationships in the factor markets in the States of Minas Gerais and São Paulo, during the period from 1975 to 2001. Specifically, it is evaluated the sensitiveness of the input use in response to price changes, as well as the interdependence with the production factor market. For that, the Allen elasticity of substitution approach was used. The input and labor expenditures presented the highest share of the average operational production costs in Minas Gerais and São Paulo. With respect to the substitutability/complementarity among the production factors in the Minas Gerais State, the result suggested that there was a complementarity relationship between land rent and machinery operations; land and labor; machinery and input uses, and a substitutability relationship among the other production factors. On the other hand, for the São Paulo State, it was observed that the variables land rent and labor had been complementary, and the other pairs were substitute factors.
\end{abstract}

Key Words: Cost, translog, Allen elasticity of substitution, coffee.

JEL classification: D24.

\title{
1 - Introdução
}

$\mathrm{O}$ processo generalizado de abertura das economias nacionais à competição internacional, vivenciado neste limiar de século, expõe empresas e setores econômicos a acirrados processos de concorrência. De acordo com o BNDES (1999), essa mudança na economia internacional tem colocado grandes desafios às empresas e países, no que se refere à manutenção de suas posições no mercado. Tendências recentes da indústria, tais como crescente ritmo de inovação tecnológica, menores ciclos de vidas dos produtos, flexibilidade no atendimento ao mercado, importância da mão-de-obra qualificada, novos insumos, mudanças 
nos padrões de organização e intensificação da competição podem implicar grandes modificações na composição e nos fluxos geográficos do comércio internacional.

PORTER (1993) enfatizou que a capacidade de um país obter padrões de vida elevados e ascendentes para seus cidadãos depende não da idéia amorfa de "competitividade", mas da produtividade com a qual os recursos nacionais (trabalho e capital) são empregados.

JOHNSTON e KILBY (1977) constataram, em seu estudo “Agricultura e transformação estrutural: estratégias econômicas de países em desenvolvimento", que existe estreita relação entre produtividade dos fatores e mudança estrutural, dado que parte significativa do aumento do produto não era explicada pelo acréscimo da quantidade dos insumos, mas pelos seus ganhos de produtividade.

Na análise das transformações estruturais da agricultura e produtividade total dos fatores no Brasil e em todas as unidades da federação, GASQUES e CONCEIÇÃO (2000) observaram que as regiões que se destacaram em direção à especialização foram as que apresentaram maiores ganhos de produtividade total dos fatores.

Face ao exposto, percebe-se a importância de estudar as vantagens competitivas dos setores econômicos por meio dos indicadores de produtividade, dado que estes estão relacionados com tecnologia de produção, que busca adaptá-los a essas tendências, e podem direcionar o acesso à competitividade, bem como a manutenção e, ou, a ampliação desta. Além disso, o estudo do processo produtivo de uma atividade é necessário para que se possa conhecer o perfil tecnológico do setor, já que este permite obter medidas que orientem a tomada de decisão, com vistas no aumento da eficiência da exploração.

A necessidade de as atividades produtivas aumentarem suas competitividades, no processo de globalização, impulsiona a modernização de suas estruturas econômicas. Com isso, mão-de-obra, capital e tecnologia movimentam-se, de forma mais livre, entre as regiões. Essa maior mobilidade dos recursos produtivos faz com que estes se concentrem em atividades que sejam mais atrativas, do ponto de vista econômico, ou seja, em atividades que propiciem melhores taxas de retorno aos investimentos.

A cafeicultura é uma atividade de exportação e vem, ao longo do tempo, contribuindo para a geração de emprego e de divisas no país. 
O potencial de crescimento da atividade cafeeira, tanto no mercado interno, em razão da estabilização, quanto no maior alcance do mercado externo, em face da globalização, é promissor diante dessa nova ordem econômica. Segundo BOUVERY (1997), somente o Brasil tem potencial para aumentar a produção em 10 ou $20 \%$, de um ano para outro, o que o coloca novamente no front das decisões sobre a atividade cafeeira. Nesse novo cenário, o setor cafeeiro brasileiro tem condições de tornar-se mais competitivo nos mercados internacional e doméstico. Esse processo de desenvolvimento da atividade cafeeira levará muitos produtores e regiões a serem marginalizados, à medida que a atividade for se profissionalizando e concentrando em regiões mais favoráveis.

Os investimentos na melhoria da qualidade e de processos representam a abertura de novos mercados para o produto. O uso de tecnologias adequadas e mais avançadas pode tornar a atividade mais competitiva. Nessa nova realidade, faz-se necessário que o produtor invista em sistemas de produção que lhe proporcionem grandes possibilidades de retorno, orientado pelas exigências do mercado consumidor.

Assim, torna-se imprescindível estudar a transformação no comportamento produtivo da cafeicultura e as relações existentes no mercado de fatores nos Estados de Minas Gerais e São Paulo, no período de 1975 a 2001. Especificamente, pretende-se avaliar a sensibilidade do uso dos insumos a alterações nos preços, mediante as elasticidades parciais de substituição de Allen, assim como a interdependência no mercado dos fatores produtivos; e verificar a existência de alterações na produtividade, quanto ao uso dos fatores de produção na cafeicultura.

\section{2 - Modelo Teórico}

O modelo utilizado neste estudo baseia-se no enfoque da dualidade entre as funções de produção e custo, a partir do qual se torna possível analisar um processo produtivo por meio de sua função custo (CHAMBERS, 1988; DEBERTIN e PAGOULATOS, 1985; e BEATTIE e TAYLOR, 1985).

Pela dualidade pode-se, sob determinadas condições, recuperar toda informação relevante sobre tecnologia de produção, a partir do estudo das funções de custo, que permite verificar as mudanças tecnológicas sem necessidade de conhecer, diretamente, a função de produção. 
A especificação de uma função de produção implica uma função de custo específica e vice-versa. Assim, a estrutura de produção pode ser estudada empiricamente, usando-se uma função de produção ou de custo. A escolha entre elas baseia-se apenas em critérios estatísticos. Por um lado, a estimação direta de uma função de produção é um procedimento atraente, nos casos em que o nível de produto é considerado endógeno; por outro, a estimação de uma função de custo, também o é, nos casos em que o nível de produto é considerado exógeno.

Nas aplicações da teoria da dualidade tem-se dado preferência pelo emprego de funções de custo por diversos fatores, a saber:

- dada a não-necessidade de se impor restrição de homogeneidade de grau um ao processo produtivo, para obtenção de equações empíricas. As funções de custo são homogêneas em preços, independentemente das propriedades de homogeneidade da função de produção, uma vez que a duplicação de todos os preços resulta em duplicação dos custos, mas não altera as razões entre quantidades de fatores;

- pelo fato de nas funções empíricas geralmente incluírem preços como variáveis explicativas, e não quantidades de fatores. Para o empresário, os preços dos fatores são, efetivamente, variáveis exógenas apropriadas, e os níveis de utilização são variáveis decisórias endógenas ao processo produtivo;

- em razão de o uso da função de custo não exigir inversão da matriz de coeficientes para que se obtenham estimativas de elasticidades de demanda, cruzada e de substituição, procedimento que pode exagerar os erros de estimação, no caso do emprego da função de produção;

- em virtude de, no caso da função de custo translog, todas as equações estimadas serem linearizadas em logaritmos;

- pelo fato de, nas equações de custo, os problemas de multicolinearidade serem menores que nas funções de produção, dada a menor correlação entre os preços dos fatores.

- pelo fato de toda função de custo implicar um conjunto de equações de demanda derivada de fatores. Além disso, diferentes formas funcionais das funções de custo desenvolvidas têm, de modo geral, resultado em equações de demanda derivada lineares nos parâmetros, representando, ao mesmo tempo, a estrutura de produção, mesmo quando não são derivadas de funções de produção explícitas. 
Diante dessas vantagens, optou-se, neste trabalho, por utilizar a função de custo em vez da de produção.

Para que se entenda melhor a função de custo, torna-se necessário definir o que é custo de produção de um produto, que é a soma de todos os pagamentos diretos e indiretos feitos pelo uso de fatores de produção (ou serviços), no processo que leva à obtenção do produto final. Este pagamento, que se caracteriza por uma despesa do produtor, é feito ao dono dos fatores de produção. Mesmo os fatores que pertencem ao proprietário do sistema de produção, como máquinas agrícolas, benfeitorias e terra, devem ser remunerados.

Uma das tarefas do produtor é selecionar determinada combinação de insumos que minimize o custo de produção, diante de ampla variedade de combinações destes, para alcançar certo nível de produção. As possíveis combinações dos insumos necessários à produção são representadas pelas isoquantas, que fornecem as restrições tecnológicas para essas combinações.

Dados os preços dos insumos empregados no processo produtivo e dado o nível de produção, são estabelecidas as linhas de isocusto, que representam as combinações de insumos que resultam no mesmo nível de custo.

O comportamento economicamente eficiente dar-se-á no ponto de tangência entre a curva de isoquanta e a linha de isocusto mais baixa possível, o qual se refere ao ponto de igualdade entre a taxa marginal de substituição dos insumos e a razão de preços dos fatores. Essa eficiência é alcançada porque nesse ponto ocorrem tanto a eficiência técnica, o máximo de produto com o mesmo fluxo de fatores fornecido pela isoquanta, quanto a eficiência-preço, dado o menor custo expresso pela linha de isocusto. Em níveis maiores de produção, novos pontos de custo mínimo são definidos. Os sucessivos pontos de tangência formam o denominado caminho de expansão da empresa, o qual expressa as despesas com os fatores, à medida que a produção vai se expandindo.

Neste trabalho, os conceitos econômicos de interesse referem-se às elasticidades-preço diretas e cruzadas da demanda dos fatores produtivos, o que permite caracterizar o processo produtivo e verificar a sensibilidade do setor a alterações nos preços dos recursos de produção. As elasticidades cruzadas da demanda permitem verificar se há interde- 
pendência no mercado de fatores, mediante relações de substituição ou complementaridade entre recursos. Essas elasticidades serão obtidas a partir dos parâmetros estruturais de um modelo de custo transcendental logarítmico (translog).

Segundo VARIAN (1984), a elasticidade de substituição entre recursos mede a variação relativa na proporção entre os fatores, causada por alterações na relação de preços desses fatores. Tal medida é válida, visto que os demais preços dos fatores produtivos são mantidos fixos e as quantidades dos fatores são ajustadas aos níveis ótimos.

A elasticidade-preço direta da demanda dos fatores indica a sensibilidade no uso dos recursos a alterações no seu preço, enquanto que a elasticidade-preço cruzada mede a reação relativa da quantidade demandada de um fator provocado pela variação no preço de outro recurso. A importância econômica desses conceitos está na possibilidade de visualizar maior ou menor independência no mercado de fatores.

Tanto a elasticidade de substituição entre fatores quanto a elasticidade cruzada da demanda permitem que se avaliem as relações de substituição ou complementaridade entre recursos. A diferença está no fato de que a primeira reflete o processo produtivo, enquanto a segunda permite que se façam inferências sobre o mercado de fatores.

A forma translog da função custo é uma forma funcional flexível, que não impõe restrições a priori à função de produção a ela associada. Em razão dessa característica, optou-se em utilizá-la neste estudo. Diferentes autores empregaram essa metodologia para analisar a função de custo, entre eles, BLACKORBY e RUSSELL (1981); ALBUQUERQUE (1987); REIS e TEIXEIRA (1995); ROCHELLE e FERREIRA FILHO (1999); e LIMA (2000).

A função custo expressa o custo de produção como função dos preços dos insumos utilizados na produção e da quantidade produzida. Admitese que, para cada quantidade produzida Y, o ponto correspondente da função de custo seja mínimo. Assim, a expressão (1) representa a função indireta de custo associada à função de produção:

$\mathrm{C}=f\left(\mathrm{Y}, \mathrm{P}_{1}, \mathrm{P}_{2}, \mathrm{P}_{3}, \ldots, \mathrm{P}_{\mathrm{N}}\right)$,

em que C é o custo mínimo para o nível de produção $\mathrm{Y}$; e $\mathrm{P}_{1}, \mathrm{P}_{2}, \mathrm{P}_{3}$, $\ldots . ., \mathrm{P}_{\mathrm{N}}$, preços dos insumos utilizados no processo produtivo. 
Considerando-se retornos constantes à escala, é possível representar a função de custo médio ou unitário por

$$
\begin{aligned}
& \mathrm{C}=\mathrm{Y} f(\mathrm{P}), \text { ou seja, } f(\mathrm{P})=\mathrm{C} / \mathrm{Y}, \\
& \mathrm{C}=f\left(\mathrm{P}_{1}, \mathrm{P}_{2}, \mathrm{P}_{3}, \ldots, \mathrm{P}_{\mathrm{N}}\right) .
\end{aligned}
$$

Aplicando-se logaritmos naturais à expressão (3) e fazendo-se a expansão por meio de uma série de Taylor em torno do vetor unitário, obtém-se a função de custo transcendental logarítmica (Translog), representada pela seguinte expressão:

$$
\ln C=\beta_{o}+\sum_{i}^{n} \beta_{i} \ln P_{i}+\frac{1}{2} \sum_{i}^{n} \sum_{j}^{n} \beta_{i j} \ln P_{i} \ln P_{j},
$$

em que os parâmetros estruturais são representados pelos $\beta$ 's, e os índices i e j identificam os fatores de produção utilizados na estimação da função custo. Para que a equação (4) satisfaça às propriedades teóricas da função de custo, ela deve ser contínua e diferenciável nos preços, obedecer às condições de simetria e homogeneidade linear (homogênea de grau um nos preços dos fatores), e, ainda, estabelecer as restrições de monotonicidade positiva e de quase concavidade em preços dos fatores (DIAS, 1982). A condição de simetria é imposta pela restrição $\beta_{i j}=\beta_{\mathrm{ji}}$, para $\mathbf{i} \neq \mathbf{j}$, e a homogeneidade linear é garantida pelas condições:

$$
\sum_{i=1}^{n} \beta_{\mathrm{i}}=1 \quad \text { e } \sum_{i=1}^{n} \beta_{i j}=\sum_{\mathrm{j}=1}^{\mathrm{n}} \beta_{\mathrm{ji}}=0 .
$$

Para a função translog, as condições de monotonicidade e concavidade são conferidas localmente. A monotonicidade da função é condicionada pelo comportamento das parcelas de custo e será atendida se estas apresentarem sinal não-negativo, já que o aumento do uso dos fatores provoca elevação dos custos. Para que a concavidade seja satisfeita, é necessário que a matriz hessiana orlada seja negativa semidefinida.

Tomando-se a derivada parcial da expressão (4), em relação a todos os preços dos fatores, e usando-se o lema de Shephard, obtém-se um sistema de $n$ equações, que representam as demandas derivadas dos fa- 
tores de produção em função dos preços. Conforme apresentado na expressão (5), a demanda de fatores é expressa pelas parcelas de custo:

$$
\frac{\partial \ln C}{\partial \ln P_{i}}=\frac{\partial C}{\partial P_{i}} \cdot \frac{P_{i}}{C}=\frac{X_{i} \cdot P_{i}}{C}=S_{i}=\beta_{i}+\sum_{j=i}^{n} \beta_{j} \ln P_{j},
$$

em que $\mathrm{X}_{\mathrm{i}}$ é a quantidade utilizada do fator i e $\mathrm{S}_{\mathrm{i}}$, a parcela de custo referente ao fator i. Assim, os parâmetros da função custo translog podem ser determinados pela estimação de $n$ parcelas de custo que compõem o sistema de equações, representado por (5).

As elasticidades de substituição parcial, de Allen, podem ser calculadas pelas expressões (8) e (9), seguindo a proposta de BISWANGER (1974).

$\sigma_{i}=\frac{\beta_{i}}{S_{i}^{2}}-\frac{1}{S_{i}}+1$

$\sigma_{\mathbf{j}}=\frac{\beta_{\mathrm{ij}}}{\mathrm{S}_{\mathrm{i}} \mathrm{S}_{\mathrm{i}}}+1$

\section{3 - Metodologia}

\section{1 - Modelo analítico}

Para analisar a mudança na produtividade da cultura de café em grãos, no período de 1975 a 2001, nos estados de Minas Gerais e São Paulo, faz-se uso da função translog de custo. Consideram-se como constituintes deste custo os seguintes itens: aluguel da terra (T), operações com máquinas (M); mão-de-obra (L) e insumos (I). Dado que a função translog de custo impõe a condição de simetria, isto é, $\beta_{\mathrm{ij}}=\beta_{\mathrm{ji}}$ para $\mathrm{i} \neq \mathrm{j}$, essa pode ser expressa por $\ln C=\beta_{0}+\beta_{T} \ln P_{T}+\beta_{L} \ln P_{L}+\beta_{I} \ln P_{I}+\beta_{M} \ln P_{M}+$ $\frac{1}{2} \beta_{T T}\left(\ln P_{T}\right)^{2}+\frac{1}{2} \beta_{T L} \ln P_{T} \ln P_{L}+\frac{1}{2} \beta_{T I} \ln P_{T} \ln P_{I}+$ $\frac{1}{2} \beta_{T M} \ln P_{T} \ln P_{M}+\frac{1}{2} \beta_{L L}\left(\ln P_{L}\right)^{2}+\frac{1}{2} \beta_{L I} \ln \mathrm{P}_{\mathrm{L}} \ln \mathrm{P}_{\mathrm{I}}+$ 


$$
\begin{aligned}
& \frac{1}{2} \beta_{L M} \ln P_{L} \ln P_{M}+\frac{1}{2} \beta_{I I}\left(\ln P_{I}\right)^{2}+\frac{1}{2} \beta_{I M} \ln P_{I} \ln P_{M}+ \\
& \frac{1}{2} \beta_{M M}\left(\ln P_{M}\right)^{2} .
\end{aligned}
$$

As equações de parcelas de custo são obtidas por meio das derivadas parciais da função translog de custo. Dessa forma, têm-se:

$S_{T}=\frac{\partial \ln C}{\partial \ln P_{T}}=\beta_{\mathrm{T}}+\beta_{T T} \ln P_{T}+\beta_{T L} \ln \mathrm{P}_{\mathrm{L}}+\beta_{T I} \ln \mathrm{P}_{\mathrm{I}}+\beta_{T M} \ln \mathrm{P}_{\mathrm{M}}$

$S_{L}=\frac{\partial \ln C}{\partial P_{L}}=\beta_{\mathrm{L}}+\beta_{T L} \ln P_{L}+\beta_{L L} \ln \mathrm{P}_{\mathrm{L}}+\beta_{L I} \ln \mathrm{P}_{\mathrm{I}}+\beta_{L M} \ln \mathrm{P}_{\mathrm{M}} ;$

$S_{I}=\frac{\partial \ln C}{\partial P_{I}}=\beta_{\mathrm{I}}+\beta_{T I} \ln P_{T}+\beta_{L I} \ln \mathrm{P}_{\mathrm{L}}+\beta_{I I} \ln \mathrm{P}_{\mathrm{I}}+\beta_{I M} \ln \mathrm{P}_{\mathrm{M}} ; \mathrm{e}$

$S_{M}=\frac{\partial \ln C}{\partial P_{M}}=\beta_{\mathrm{M}}+\beta_{T M} \ln P_{T}+\beta_{L M} \ln \mathrm{P}_{\mathrm{L}}+\beta_{M I} \ln \mathrm{P}_{\mathrm{I}}+\beta_{M M} \ln \mathrm{P}_{\mathrm{M}}$

As equações de parcelas de custo, expressas pelas equações (10), são estimadas pelo método iterativo de Zellner, que objetiva estimar parâmetros de equações aparentemente não-relacionadas (SUR), uma vez que possuem ligações por meio dos seus erros aleatórios. Para tornar o modelo operacional são estimadas $n-1$ equações de parcelas de custo, com o intuito de evitar uma matriz singular de covariância dos erros, uma vez que a soma das parcelas é igual a um.

Cabe destacar ainda, no procedimento para estimação de Zellner, que o resultado é invariante em relação à equação suprimida (KMENTA, 1990).

Para captar o comportamento das alterações tecnológicas dos custos de produção foram incluídas duas variáveis dummies, $\left(\mathrm{D}_{1}\right.$ e $\left.\mathrm{D}_{2}\right)$ consideradas como "fixas" no modelo, em razão do comportamento dos seus valores no intervalo de tempo entre 1975-79, 1980-89 e 1990 a 2001.

Espera-se que essas variáveis forneçam subsídios para se analisar o comportamento das alterações tecnológicas dos componentes dos custos de produção nos subperíodos de análise.

O subperíodo de 1975-79 foi considerado como base, atribuindo-se 
o valor zero para as duas variáveis binárias $\left(D_{1}=0\right.$ e $\left.D_{2}=0\right)$. No subperíodo 1980-89 atribuíram-se as variáveis dummies $\mathrm{D}_{1}=1$ e $\mathrm{D}_{2}=0$, e no de 1990 a $2001, D_{1}=0$ e $D_{2}=1$.

\section{2 - Fonte de dados}

Os dados utilizados neste estudo foram as séries de Custo Operacional Total (COT) de produção, levantados, anualmente, por uma cooperativa de Cafeicultores do Sul de Minas Gerais. Para São Paulo, utilizaram-se os custos definidos por MELLO et. al. (1978), BESSA JUNIOR et al. (1979), MELLO et. al. (1985), AGRIANUAL (2000) e AGRIANUAL (2001). Os preços usados foram obtidos na publicação Informações Econômicas ( vários volumes) do Instituto de Economia Agrícola (IEA).

Todos os valores e preços, no período de 1975 a 2001, foram atualizados para maio de 2002, pelo Índice Geral de Preços Disponibilidade Interna (IGP-DI), da Fundação Getúlio Vargas - FGV (2002).

O Custo Operacional Total (COT) de produção, por hectare, pode ser definido pelo Custo Operacional Efetivo (COE) mais depreciação e juros bancários. O Custo Operacional Efetivo é composto pelas despesas com operações mais o material consumido. Nas despesas com operações incluem-se mão-de-obra comum, mão-de-obra utilizada na colheita, trator, fertilizantes, herbicidas, inseticidas.

O sistema de produção de café adotado foi de 1.000 a 1.666 covas por hectares, com uma produtividade de 11,44 sacas a 30 sacas de café beneficiado por hectare.

Os fatores de produção considerados foram: terra (T), mão-de-obra (L) e operações com máquinas (M) e insumos (I). As parcelas de custo dos fatores de produção são representadas pelas despesas totais com cada um deles. Nas despesas com serviços foram consideradas a mão-de-obra comum (adubação do solo, aplicação de defensivos, capinas manuais, desbrotas, arruação, aplicação de calcário, esparramação, pré-secagem, secagem, beneficiamento) e mão-de-obra para colheita.

Nas despesas com operações com máquinas foram consideradas a hora-trator, o beneficiamento e a secagem, e nos dispêndios com insumos, fertilizantes e defensivos agrícolas. 


\section{4 - Resultados e Discussão}

Nesta parte discutem-se os resultados do modelo econométrico da função translog de custo, estimada para a cafeicultura nos Estados de Minas Gerais e São Paulo, e, a partir dos resultados obtidos por meio da taxa geométrica de crescimento (TGC), são analisadas as mudanças na produtividade, nos custos operacionais unitários e nas parcelas de custos dos fatores da cafeicultura em ambas as regiões.

\section{1 - Análise das equações das parcelas de custo operacional unitário de café para o Estado de Minas Gerais}

De acordo com Hunt e Lynk (1993) e Syern (1995), citados por LIMA (2000), é difícil a interpretação dos coeficientes estimados (sinal e magnitude) pelo modelo translog e por outras funções que envolvem termos logarítmicos multiplicativos, em face da dependência das unidades de medida das variáveis envolvidas. Muitos autores indicam que esses coeficientes não têm significado econômico, o que não é correto. Para fazer a interpretação, tem-se que realizar transformações das unidades de medida. As elasticidades são invariantes em relação às unidades de medidas, razão por que são analisadas e interpretadas.

As estimativas das parcelas de custo foram obtidas por meio do software Eviews, utilizando-se o método de equações aparentemente nãorelacionadas (SUR), com imposição das restrições de homogeneidade linear e simetria ao modelo.

A homogeneidade linear foi garantida pelo somatório igual à unidade da coluna, referente aos interceptos das equações das parcelas de custo $\left(\sum_{i=1}^{n} \beta_{i}=1\right)$, e pelo somatório, igual a zero, das demais colunas e de todas as linhas da tabela $\left(\sum_{i=1}^{n} \beta_{i j}=\sum_{j=1}^{n} \beta_{i j}=0\right)$. A condição de simetria foi imposta

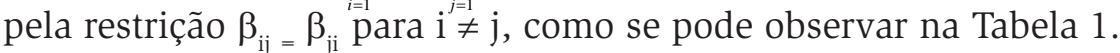

O modelo estimado para Minas Gerais (Tabela 1) apresentou bom ajustamento da função aos dados, uma vez que, em todas as equações de parcelas, os coeficientes de determinação $\left(\mathrm{R}^{2}\right)$ ficaram acima de $80 \%$.

Os coeficientes $\beta_{\mathrm{LM}}(-0,023781)$ e $\beta_{\mathrm{ML}}(-0,023781)$ foram os únicos que não foram estatisticamente significativos; $\beta_{\mathrm{LM}}$ é o coeficiente que representa a relação entre a variável operações com máquinas na equação de 
parcela de custo referente à mão-de-obra e $\beta_{\mathrm{ML}}$, coeficiente que representa a relação entre a variável mão-de-obra na equação de parcela de custo referente a operações com máquinas (Tabela 1).

A monotonicidade da função foi garantida pelo comportamento das parcelas de custo, que foram positivas e calculadas pela média aritmética da amostra, obtendo-se os seguintes valores: $\mathrm{ST}=0,06$; $\mathrm{SL}=0,38$; SI $=0,42$ e $\mathrm{SM}=0,13$. Pode-se verificar que a maior parcela se refere às despesas com insumos. A participação dos insumos é bastante expressiva, provavelmente em razão das exigências do processo produtivo de café, uma vez que essa atividade requer maiores cuidados para que se obtenha boa produção.

As variáveis dummies $\mathrm{D}_{1 \text { e }} \mathrm{D}_{2}$ são utilizadas neste trabalho para detectar alterações no comportamento da produção cafeeira nas regiões analisadas.

Em Minas Gerais, como se pode observar, as variáveis dummies $\mathrm{D}_{1}$ e $\mathrm{D}_{2}$ evidenciam mudanças no comportamento da produtividade na cafeicultura, nos períodos de análise.

Tabela 1 - Resultados da estimativa das equações parciais de custo operacional unitário de café no Estado de Minas Gerais, 1975-2001

\begin{tabular}{|c|c|c|c|c|c|c|c|c|}
\hline Parcelas & Intercepto & $\beta_{\mathrm{T}}$ & $\beta_{\mathrm{L}}$ & $\beta_{I}$ & $\beta_{M}$ & $\mathrm{D}_{1}$ & $\mathrm{D}_{2}$ & Soma \\
\hline ST & $\begin{array}{c}-0,226016 \\
(-5,03107)^{* * *}\end{array}$ & $\begin{array}{c}0,085383 \\
(14,78909)^{* * *}\end{array}$ & $\begin{array}{c}-0,048601 \\
(-4,92606)^{* * *}\end{array}$ & $\begin{array}{c}-0,022376 \\
(-2,23396)^{* *}\end{array}$ & $\begin{array}{c}-0,014867 \\
(-1,95292)^{*}\end{array}$ & $\begin{array}{c}-0,021844 \\
(-2,91639)^{* * *}\end{array}$ & $\begin{array}{c}-0,029442 \\
(-3,60295)^{* * *}\end{array}$ & 0 \\
\hline SL & $\begin{array}{l}0,1767748 \\
(1,88450)^{*}\end{array}$ & $\begin{array}{c}-0,048601 \\
(-4,92606)^{* * *}\end{array}$ & $\begin{array}{c}0,2000448 \\
(6,26182)^{* * *}\end{array}$ & $\begin{array}{c}-0,12590 \\
(-4,8207)^{* * *}\end{array}$ & $\begin{array}{l}-0,023781 \\
(-1,2779)^{\mathrm{ns}}\end{array}$ & $\begin{array}{l}-0,132186 \\
(-7,0161)^{* * *}\end{array}$ & $\begin{array}{l}0,096845 \\
(4,2106)^{* * *}\end{array}$ & 0 \\
\hline SI & $\begin{array}{c}1,042553 \\
(7,7072)^{* * *}\end{array}$ & $\begin{array}{c}-0,022376 \\
(-2,2339)^{* *}\end{array}$ & $\begin{array}{c}-0,12590 \\
(-4,8207)^{* * *}\end{array}$ & $\begin{array}{c}0,209873 \\
(5,8260)^{* * *}\end{array}$ & $\begin{array}{c}-0,064082 \\
(-3,6047)^{* * *}\end{array}$ & $\begin{array}{c}0,229918 \\
(9,2457)^{* * *}\end{array}$ & $\begin{array}{l}0,071535 \\
(2,6815)^{* *}\end{array}$ & 0 \\
\hline SM & $\begin{array}{c}-0,003717 \\
(-0,0528)^{\mathrm{ns}}\end{array}$ & $\begin{array}{l}-0,014867 \\
(-1,9529)^{* *}\end{array}$ & $\begin{array}{c}-0,023781 \\
(-1,2779)^{\mathrm{ns}}\end{array}$ & $\begin{array}{c}-0,064082 \\
(-3,6047)^{* * *}\end{array}$ & $\begin{array}{c}0,100760 \\
(5,960182)\end{array}$ & $\begin{array}{l}-0,074997 \\
(-5,7218)^{* * *}\end{array}$ & $\begin{array}{c}-0,140333 \\
(-9,3082)^{* * *}\end{array}$ & 0 \\
\hline Soma & 1 & 0 & 0 & 0 & 0 & 0 & 0 & \\
\hline \multicolumn{9}{|c|}{$\begin{array}{l}\text { Fonte: Dados da pesquisa. } \\
* * * \text { Estatisticamente significativo a } 1 \% ; * * \text { estatisticamente significativo a } 5 \% ; \\
\text { * estatisticamente significativo a } 10 \% ; \mathrm{ns}=\text { não-significativo. } \\
\mathrm{ST}=\text { parcela de custo referente a aluguel da terra; } \mathrm{SL}=\text { parcela de custo referente à mão-de-obra; } \\
\mathrm{SI}=\text { parcela de custo referente a insumos; } \mathrm{SM}=\text { parcela de custo referente às operações com } \\
\text { máquinas. Os } \beta \text { 's foram obtidos por meio da variável } \ln (\mathrm{Pi} / \mathrm{Pj}) \text {, conforme modelo descrito. }\end{array}$} \\
\hline
\end{tabular}


A variável $\mathrm{D}_{1}$ foi significativa nas equações de parcelas de custos (aluguel da terra, mão-de-obra, operações com máquinas e insumos), o que indica que, no subperíodo de 1980 a 1989, essa função se deslocou, em relação ao subperíodo de 1975 a 1979. Com relação às parcelas aluguel da terra, mão-de-obra e operações com máquinas, constata-se que a proporção de despesa dessas parcelas no custo operacional unitário foi menor nesse subperíodo do que no período-base, já na parcela de insumos se verificou aumento de 0,2299, o que significa que a respectiva participação no custo total foi, em média, maior nesse subperíodo do que no base, o que pode estar relacionado com a produção e com o preço do café, nos dois sub-períodos.

Em relação à variável $\mathrm{D}_{2}$, observa-se que o seu coeficiente foi significativo em todas as equações de parcelas de custos, o que demonstra que, de 1990 a 2001, houve considerável aumento na produção cafeeira de Minas Gerais, acarretado pelos incentivos do governo federal que, a partir da geada de 1975, intensificou a recuperação e a diversificação do parque cafeeiro. Isto resultou na queda real dos preços do café, com conseqüente diminuição na renda dos cafeicultores, o que os levou a reduzir a utilização de terra e operações com máquinas.

O coeficiente da variável binária $\mathrm{D}_{2}$ permite concluir que as equações de parcela de custo, atribuídas a mão-de-obra e insumos, deslocaramse para cima no subperíodo de 1990 a 2001, quando comparadas às do período-base (1975-1979), o que indica que, em média, a proporção das despesas com essas parcelas foi maior nesse período. O aumento na parcela de mão-de-obra, durante o subperíodo de 1990 a 2001, pode ser explicado quando se considera a mão-de-obra como fator substituto de operações com máquinas. Como o preço desse fator estava relativamente caro nesse período, foi necessária a utilização de maior contingente de pessoas para suprir a carência do fator operações com máquinas. Em relação ao fator aluguel da terra, percebe-se, no Tabela 1, que este também apresentou redução em sua parcela de participação.

\subsection{2 - Características do processo produtivo da cafeicultura em Minas Gerais}

A característica do processo produtivo da cafeicultura é analisada pela elasticidade de substituição, de Allen, a qual relaciona a variação 
no preço relativo de um fator com a respectiva variação na quantidade utilizada do outro fator em questão.

A análise dos valores das elasticidades de substituição, apresentados no Tabela 2, evidencia a intensidade de uso entre recursos, o que reflete substitutibilidade ou complementaridade no processo de produção. As elasticidades de substituição são simétricas, e as apresentadas ao longo da diagonal principal têm pouco significado econômico.

O Tabela 2 apresenta os resultados das elasticidades de substituição parcial, de Allen, entre fatores. O sinal positivo do coeficiente indica substituição entre fatores, enquanto o negativo indica complementaridade entre eles. Conforme esperado pela teoria, todos os valores na diagonal principal apresentaram sinais negativos, com exceção do fator terra, cujo sinal foi positivo. Embora tenha pouco significado econômico, esse resultado indica que todo fator de produção é complementar a si próprio, embora o fator terra tenha dada relação de substitutibilidade com si próprio.

De acordo com o conceito de elasticidade de substituição parcial, o fator terra foi complementar em relação a operações com máquinas $\left(\sigma_{\mathrm{TM}}=0,674\right)$ e mão-de-obra $\left(\sigma_{\mathrm{TL}}=1,01\right)$, na produção de café. Essas elasticidades mostram que os cafeicultores utilizam processos de produção que favorecem a complementaridade desses fatores. Variação de $10 \%$ no preço relativo dos fatores de operações com máquinas e mão-de-obra reduzem em $6,74 \%$ e $10,10 \%$ a quantidade utilizada de terra no processo produtivo, respectivamente.

Os fatores operações com máquinas e mão-de-obra foram substitutos na produção de café. Aumento relativo (redução) de $10 \%$ no preço da mão-de-obra implica que a quantidade utilizada de operações com máquinas aumentará (reduzirá) 5,3\% . O mesmo é válido para a elasticidade de substituição, de Allen, em operações com máquinas e mão-de-obra, uma vez que elas são simétricas (Tabela 2). 
Tabela 2 - Estimativa das elasticidades de substituição parcial, de Allen, diretas e cruzadas, entre fatores para produção de café no Sul de Minas, Estado de Minas Gerais (1975-2001)

\begin{tabular}{lcccc}
\hline & Aluguel terra & Op. Máquinas & Mão-de-obra & Insumos \\
Aluguel da terra & 6,0419 & $-0,6744$ & $-1,0101$ & 0,1784 \\
Op. máquinas & $-0,9501$ & 0,5309 & $-0,0669$ \\
Mão-de-obra & & $-0,2625$ & 0,2215 \\
Insumos & & & $-0,1907$ \\
\hline
\end{tabular}

Fonte: Dados de pesquisa.

Por sua vez, os fatores operações com máquinas e insumos foram complementares.

Em relação à mão-de-obra e insumos, estes são considerados substitutos no processo produtivo. Nesse caso, quando o preço relativo de um fator aumentar (diminuir) 10\%, a quantidade utilizada do fator substituto na produção de café aumentará (diminuirá) 2,2\%, ou seja, haverá aumento (ou redução) na quantidade utilizada de um fator menos que proporcional ao aumento (redução) do preço do fator substituto.

Os resultados da Tabela 2 demonstram fraca relação de substitutibilidade e complementaridade dos fatores, o que indica que a variação na demanda de um fator é menos que proporcional à variação no preço relativo entre eles, com exceção da elasticidade de substituição, de Allen, entre preço da terra e mão-de-obra, que foi maior que a unidade $\left(\sigma_{\mathrm{TL}}=\right.$ -1,01). Esse resultado mostra que há relação de complementaridade bem próxima de um, ou seja, aumento de $10 \%$ no preço da terra reduzirá a quantidade utilizada de mão-de-obra em $10,1 \%$, e vice-versa.

Cabe destacar, ainda, que os valores das elasticidades de substituição entre terra e insumos, operações com máquinas e insumos, mão-de-obra e insumos sinalizam que os pares desses recursos são empregados em proporções fixas nas propriedades de café em Minas Gerais. 


\section{2 - Estrutura de demanda dos fatores de produção da cafeicultura em São Paulo}

O modelo estimado apresentou bom ajustamento da função aos dados, com comportamento satisfatório dos resíduos. Os coeficientes das variáveis dummies, D1 e D2, e $\beta_{\mathrm{TM}}$ para parcela de terra (ST); o intercepto para parcela de mão-de-obra (SL); e o coeficiente $\beta_{\mathrm{MT}}$ para parcela de máquinas (SM) foram não-significativos. Vale ressaltar ainda que, em todas as equações de parcelas, o $\mathrm{R}^{2}$ estimado ficou acima de $80 \%$ (Tabela 3 ).

A monotonicidade da função foi garantida pelo comportamento das parcelas de custo, que foram positivas e tiveram os seguintes valores: $\mathrm{ST}=0,10, \mathrm{SL}=0,30, \mathrm{SI}=0,41$ e $\mathrm{SM}=0,18$. Observa-se que, de modo similar a Minas Gerais, a maior parcela é de despesas com insumos, em face das exigências requeridas desse insumo na produção de café, para que se obtenha boa produção.

Quanto às variáveis dummies, como estas não foram significativas na equação de parcela de terra, pode-se inferir que não houve alteração no comportamento destas nos subperíodos de 1980-89 e 1990-2001, em relação ao subperíodo base (Tabela 3).

No que tange à parcela de insumos, observa-se que, nos subperíodos de 1980-89 e 1990-2001, houve aumento de 0,16 e 0,31, respectivamente, fato que pode estar relacionado com tratos culturais e preço do café.

Tabela 3 - Resultados da estimativa das equações parciais de custo operacional unitário de café no Estado de São Paulo, 1975-2001

\begin{tabular}{|c|c|c|c|c|c|c|c|c|}
\hline Parcelas & Intercepto & $\beta_{\mathrm{T}}$ & $\beta_{\mathrm{L}}$ & $\beta_{1}$ & $\beta_{\mathrm{M}}$ & D1 & D2 & Soma \\
\hline ST & $\begin{array}{c}-0.2394 \\
(-3.1547)^{* * *}\end{array}$ & $\begin{array}{c}0.0787 \\
(8.1469)^{* * *}\end{array}$ & $\begin{array}{c}-0.0372 \\
(-3.3714)^{* * *}\end{array}$ & $\begin{array}{c}-0.0392 \\
(-2.1931)^{* *}\end{array}$ & $\begin{array}{c}-0.0024 \\
(-0.3212)^{\mathrm{NS}}\end{array}$ & $\begin{array}{c}0.0049 \\
(0.2202)^{\mathrm{NS}}\end{array}$ & $\begin{array}{c}-0,0220 \\
(-1.1582)^{\mathrm{NS}}\end{array}$ & 0 \\
\hline SL & $\begin{array}{c}0.0531 \\
(0.4445)^{\mathrm{NS}}\end{array}$ & $\begin{array}{c}-0.0372 \\
(-3.3714)^{* * *}\end{array}$ & $\begin{array}{c}0.1744 \\
(8.1516)^{* * *}\end{array}$ & $\begin{array}{c}-0.0862 \\
(-2.7574)^{* * *}\end{array}$ & $\begin{array}{c}-0.0486 \\
(-4.0120)^{* * *}\end{array}$ & $\begin{array}{c}0.1040 \\
(2.9836)^{* * *}\end{array}$ & $\begin{array}{c}0.0537 \\
(1.9525)^{*}\end{array}$ & 0 \\
\hline SI & $\begin{array}{c}0.7929 \\
(3.6069)^{* * *}\end{array}$ & $\begin{array}{c}-0.0392 \\
(-2.1931)^{* *}\end{array}$ & $\begin{array}{c}-0.0862 \\
(-2.7574)^{* * *}\end{array}$ & $\begin{array}{c}0.1648 \\
(2.9510)^{* * *}\end{array}$ & $\begin{array}{c}-0.0429 \\
(-2.0169)^{* *}\end{array}$ & $\begin{array}{c}0.1601 \\
(2.4614)^{* *}\end{array}$ & $\begin{array}{c}0.3100 \\
(6,5882)^{* * *}\end{array}$ & 0 \\
\hline SM & $\begin{array}{c}0.3809 \\
(4.6732)^{* * *}\end{array}$ & $\begin{array}{c}-0.0024 \\
(-0.3212)^{\mathrm{NS}}\end{array}$ & $\begin{array}{c}-0.0486 \\
(-4.0120)^{* * *}\end{array}$ & $\begin{array}{c}-0.0429 \\
(-2.0169)^{* *}\end{array}$ & $\begin{array}{c}0.0936 \\
(7.1778)^{* * *}\end{array}$ & $\begin{array}{c}-0.2663 \\
(-8.4236)^{* * *}\end{array}$ & $\begin{array}{c}-0.3403 \\
(-13.4873)^{* * *}\end{array}$ & 0 \\
\hline Soma & 1 & 0 & 0 & 0 & 0 & 0 & 0 & \\
\hline
\end{tabular}

Fonte: Dados da pesquisa.

*** Estatisticamente significativo a 1\%; * estatisticamente significativo a 5\%; * estatisticamente significativo a $10 \%$; ns = não-significativo. 
$\mathrm{ST}=$ parcela de custo referente a aluguel da terra; $\mathrm{SL}=$ parcela de custo referente à mão-de-obra; $\mathrm{SI}=$ parcela de custo referente a insumos; $\mathrm{SM}=$ parcela de custo referente às operações com máquinas. Os $\beta$ ’s foram obtidos por meio da variável $\ln (\mathrm{Pi} / \mathrm{Pj})$, conforme modelo descrito.

A parcela de mão-de-obra obteve respectivos aumentos de 0,10 e 0,05, em relação ao subperíodo-base, nos subperíodos de 1980-89 e 19902001. Já na parcela operações com máquinas houve redução na ordem de 0,27 e 0,34, o que pode ser explicado pelo fato de o fator operações com máquinas, nesses subperíodos, ter sido relativamente mais caro do que o fator mão-de-obra, o que provocou aumento na quantidade de pessoas contratadas para suprir a carência de operações com máquinas, como ocorreu em Minas Gerais.

\subsection{1 - Características do processo produtivo no Estado de São Paulo}

Conforme resultados apresentados na Tabela 4, a elasticidade de substituição, de Allen, mostra coerência entre as estimativas das elasticidades-cruzadas nos diferentes fatores de produção. A elasticidade entre aluguel da terra e mão-de-obra foi negativa, o que indica complementaridade entre esses fatores, enquanto entre outros pares esta apresentou sinal positivo, o que retrata que estes são substitutos entre si.

Tabela 4 - Estimativa das elasticidades de substituição parcial, diretas e cruzadas, entre fatores de produção de café no Estado de São Paulo, 1975-2001

\begin{tabular}{lcccc}
\hline & Aluguel terra & Op. Máquinas & Mão-de-obra & Insumos \\
Aluguel terra & -1.2803 & 0.8692 & -0.1933 & 0.0761 \\
OP. Máquinas & & -1.6684 & 0.1159 & 0.4251 \\
Mão-de-obra & & -0.4015 & 0.3111 \\
Insumos & & & -0.4559 \\
\hline
\end{tabular}

Fonte: Dados da pesquisa.

Cabe destacar, ainda, que essas elasticidades são simétricas e do tipo "um fator - um preço", ou seja, mostram a mudança percentual na quantidade de um fator, resultante da mudança percentual no preço do outro fator. É uma medida de como um fator se ajusta a mudanças no preço relativo do outro fator. A elasticidade de substituição de aluguel da terra e operações com máquinas (ou vice-versa ) foi de 0,87 , o que indica que elevação de $10 \%$ no preço de operações com máquinas causa aumento 
de $8,7 \%$ na quantidade utilizada de terra. Da mesma forma, devido à simetria, incremento de $10 \%$ no preço do aluguel da terra provoca aumento de 8,7\% na quantidade usada com operações com máquinas. Essa mesma relação é válida para os demais pares de valores, que apresentaram sinal positivo. Ressalta-se ainda que a relação de substitutibilidade mais elevada foi encontrada entre o fator terra e operações com máquinas no processo de produção de café no Estado de São Paulo.

A relação de complementaridade entre os fatores aluguel da terra e mão-de-obra mostra que aumento de $10 \%$ no preço do aluguel da terra reduz em 1,9\% a quantidade usada de mão-de-obra no processo de produção de café no Estado de São Paulo.

\section{3 - Mudanças na produtividade e nos custos da cafeicultura em Minas Gerais e São Paulo}

A cafeicultura é uma cultura que é inviável de ser explorada com baixos rendimentos, visto que seus retornos econômicos estão estritamente vinculados à produtividade. Por ser uma cultura que exige tratos culturais e requer grandes inversões fixas, possibilita retornos compensadores quando produzida com altas produtividades.

Os custos unitários de produção de café (saca de 60 kg) em Minas Gerais e São Paulo, no período de 1975 a 2001, apresentaram variações negativas de 4,25\% e 4,50 \% ao ano, enquanto as produtividades (saca de $60 \mathrm{~kg}$ /hectare) apresentaram variações positivas de 3,78\% e 3,52\% ao ano, respectivamente (Tabela 5). Assim, pode-se constatar que os custos decresceram à medida que as produtividades aumentaram, e o aumento da produtividade foi maior em Minas Gerais do que em São Paulo.

Esse crescimento da produtividade da cafeicultura, nesses estados, pode estar relacionado com as mudanças que ocorreram na economia nesse período, isto é, redução no crédito agropecuário, extinção do IBC, rompimento dos acordos internacionais do café e maior abertura da economia, o que levou os produtores a aumentarem a produtividade e reduzirem os custos de produção por unidade. 
Tabela 5 - Taxas anuais de crescimento da produtividade, do custo unitário e das parcelas de participação dos fatores nos custos unitários (saca de café de 60 kg) nos Estados de Minas Gerais e São Paulo, 1975-2001

\begin{tabular}{lcc}
\hline & Minas Gerais & São Paulo \\
\hline Produtividade & 3,78 & 3,52 \\
Custo & -4.25 & -4.50 \\
Terra & $-1,36$ & $-0,93$ \\
Mão-de-obra & 1,65 & 1,79 \\
Operações com máquinas & $-4,47$ & $-6,85$ \\
Insumos & 0,62 & 0,34 \\
\hline
\end{tabular}

Fonte: Dados da pesquisa.

Em Minas Gerais, o custo unitário de produção de 11,44 sacas de café foi 2,48 vezes maior do que o de produção de 30 sacas por hectare. Já no Estado de São Paulo, o custo unitário de produção de 12,2 sacas foi 3,22 vezes maior do que o de 30 sacas por hectares.

Com base nas taxas de crescimento das parcelas de custo dos fatores de produção, no período de 1975 a 2001, em Minas Gerais e São Paulo, podem-se notar aumento das parcelas de mão-de-obra e insumos e queda das parcelas dos fatores terra e operações com máquinas, à medida que aumentava a produtividade (Tabela 5). Dessa forma, utilização de maiores quantidades de mão-de-obra e insumos por hectare cultivado de café, associada aos demais fatores acima citados, pode ter contribuído para o aumento da produtividade, ao longo do período de análise.

\section{5 - Conclusões}

Os resultados permitem concluir que as despesas com insumos e mão-de-obra representam as maiores parcelas dos custos operacionais unitários de produção em Minas Gerais e São Paulo.

As variáveis dummies utilizadas mostram mudanças ocorridas nas parcelas de participação dos fatores de produção no custo unitário, entre os subperíodos, nos Estados de Minas Gerais e São Paulo. Nota-se que os produtores de café reduziram a quantidade de operações com máquinas e aumentaram o uso de mão-de-obra e insumos, enquanto a utilização de terra manteve-se, praticamente, inalterada durante o período analisado. 
No que concerne às elasticidades de substituição parcial para os fatores aluguel da terra, operações com máquinas, mão-de-obra e insumos, utilizados na produção de café no período de 1975 a 2001, verifica-se, no Estado de São Paulo, comportamento coerente com a teoria econômica, uma vez que as elasticidades diretas de substituição apresentaram sinal negativo. Entretanto, no Estado de Minas Gerais, o fator terra foi o único que apresentou sinal contrário à teoria.

Em relação à substitutibilidade/complementaridade entre os fatores em Minas Gerais, nota-se que existe relação de complementaridade entre aluguel da terra e operações com máquinas, aluguel da terra e mão-deobra, operações com máquinas e insumos. Contudo, entre os demais pares de fatores, a relação foi de substituição. A magnitude da elasticidade de substituição parcial, de Allen, entre mão-de-obra e operações com máquinas permite concluir que a variação nos preços desses fatores afetou, sensivelmente, a composição dos custos de produção de café. Já no Estado de São Paulo, os fatores aluguel da terra e mão-de-obra foram complementares, e os demais, substitutos.

Em face dos resultados obtidos, sugere-se que os cafeicultores procurem conhecer melhor as relações existentes entre os fatores de produção utilizados na atividade, para que, em razão das alterações nos preços dos mesmos, possam definir ações que visem alocar mais eficientemente os recursos produtivos, com vistas na ampliação de maior eficiência na exploração e maior produtividade, melhor qualidade e menor custo de produção.

\section{6 - Referências bibliográficas}

AGRIANUAL. Anuário Estatístico da Agricultura Brasileira. São Paulo: FNP Consultoria e Comércio, 2000.

AGRIANUAL. Anuário Estatístico da Agricultura Brasileira. São Paulo: FNP Consultoria e Comércio, 2001.

ALBUQUERQUE, M. C. C. DE. Uma análise Translog sobre mudança tecnológica e efeitos de escala: um caso de modernização ineficiente. Pesquisa e planejamento econômico, Rio de Janeiro, v.17, n.1, p.191-220, abr.1987. 
BANCO NACIONAL DE DESENVOLVIMENTO ECONÔMICO E SOCIALBNDS. Competitividade: conceituação e fatores determinantes. Rio de Janeiro: BNDS; mar./99. p. 26 (Textos para discussão, 2).

BEATTIE, B.R., TAYLOR, C. R. The economics of production. New York: John Eiley e Sons, 1985, 258p.

BESSA JUNIOR, A. de A. B. et. al..; Estimativa de Custo operacional e coeficientes técnicos das principais explorações agropecuárias, Estado de São Paulo, 1979/80. Informações econômicas. São Paulo, v. 9, n. 7, p. 17- 114, julho de 1979.

BINSWANGER, H. P. A cost function approach to the measurement of elasticities of sustitution. American journal of agricultural economics, Lexington, v. 56, n. 2, p. 377-386, May 1974.

BLACKORBY, C. e RUSSELL, R. R. The Morishima elasticity of substitution; symmetry, constancy, separability, and its relationship to the Hicks and Allen elasticities. Review of economic studies, v.48, p.147-158, 1981.

BOUVERY, B. Brasil: quem sabe? Anuário estatístico do café - Coffee Business, Rio de Janeiro, 1997. p. 3-8.

CHAMBERS, R. Applied production analysis: a dual approach. Cambridge University Press, 1988. 331p.

DEBERTIN, D. L. ; PAGOULATOS, A. Contemporary production thery, duality, elasticities of substitution, the translog production function and agricultural research. Agricultural Economics Research Report, University of Kentucky, v. 40, june 1985.

DIAS, R. S. Elasticidade de substituição e de demanda de fatores na agricultura brasileira. Viçosa: UFV, 1982. 58p. Dissertação (Mestrado Economia Rural) - Universidade Federal de viçosa, 1982.

FUNDAÇÃO GETÚLIO VARGAS - FGV. fgvdados. Disponível em <http// www.fgvdados.fgv.br> acesso em mar., 2002.

GASQUES, J. G.; CONCEIÇÃO, J. C. P. R. da. Transformações estruturais da agricultura e produtividade total dos fatores. Texto para discussão n.768. Brasília, 2000. 
INSTITUTO DE ECONOMIAAGRICOLA - IEA. Informações econômicas. São Paulo, vários volumes.

JOHNSTON, B. F., KILBY, P. Agricultura e transformação estrutural: estratégias econômicas de Países em Desenvolvimento. Rio de Janeiro: Zahar, 1977. 458 p.

LIMA, J. E. de. Definições de elasticidades de substituição: Revisão e aplicação. Revista brasileira de economia e sociologia rural, Brasília, v.38,n.1, p. 9-44. Jan./mar.2000.

MELLO, N. T.C., et al.; Estimativa de Custo operacional e coeficientes técnicos das principais explorações agropecuárias , Estado de São Paulo. Informações econômicas. São Paulo, v. 8, n. 7, p. 01-97, julho de 1978.

MELLO, N. T.C., et al.; Custo de Produção: Estimativas e coeficientes técnicos das principais atividades agropecuárias do Estado de São Paulo, safra 1985/86. Informações econômicas. São Paulo, v. 15, n. 7, p. 19-116, julho de 1985 .

KMENTA, J. Elementos de econometria: Teoria econométrica básica. São Paulo: Atlas, 1990. v.2, 711p.

PORTER, Michael E. A vantagem competitiva das nações. Editora Campus, Rio de Janeiro, 1993. 897p.

REIS, R.P.; TEIXEIRA, E. C. Estrutura de demanda e substituição de fatores produtivos na pecuária leiteira: O modelo de custo translog. Revista brasileira de economia e sociologia rural, Rio de Janeiro, v.49,n.3, p. 545-554, jul.set. 1995.

ROCHELEE, T. C. P.; FERREIRA FILHO, J. B. de S. Função de Custo Translog e o mercado de fatores para o algodão no estado de São Paulo: O caso da Dira de Campinas, Revista de economia e sociologia rural, Brasília, V.37, n.2, p. 77-95, Abr./Jun. 1999.

VARIAN, H.R., Microeconomic analysis. 2 ed. New York: Norton, 1984.

Recebido em agosto de 2004 e revisto em novembro de 2005 